This item was submitted to Loughborough's Research Repository by the author.

Items in Figshare are protected by copyright, with all rights reserved, unless otherwise indicated.

Mesoscopic Josephson arrays interacting with non-classical electromagnetic fields and their applications

PLEASE CITE THE PUBLISHED VERSION

http://dx.doi.org/10.1049/ip-smt:20010394

PUBLISHER

(C) IEEE

VERSION

VoR (Version of Record)

LICENCE

CC BY-NC-ND 4.0

REPOSITORY RECORD

Konstadopoulou, A., J.M. Hollingworth, Mark J. Everitt, A. Vourdas, T.D. Clark, and J.F. Ralph. 2019.

"Mesoscopic Josephson Arrays Interacting with Non-classical Electromagnetic Fields and Their Applications". figshare. https://hdl.handle.net/2134/12073. 
This item was submitted to Loughborough's Institutional Repository (https://dspace.lboro.ac.uk/) by the author and is made available under the following Creative Commons Licence conditions.

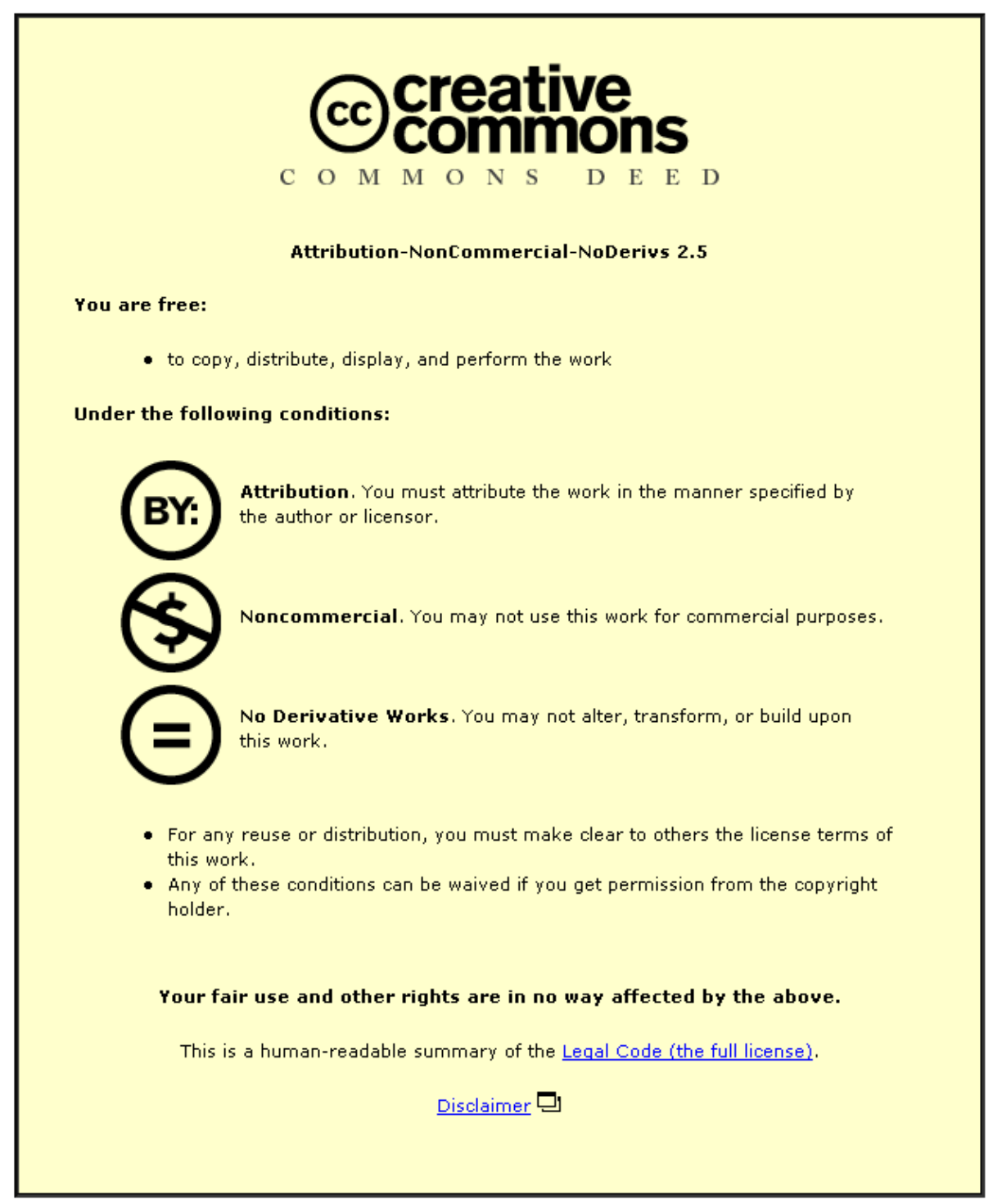

For the full text of this licence, please go to: http://creativecommons.org/licenses/by-nc-nd/2.5/ 


\title{
Mesoscopic Josephson arrays interacting with non-classical electromagnetic fields and their applications
}

\author{
A.Konstadopoulou, J.M.Hollingworth, M.Everitt, A.Vourdas, T.D.Clark and J.F.Ralph
}

\begin{abstract}
A ring made from a Josephson array in the insulating phase is considered. The ring contains a 'dual Josephson junction' (Josephson junction for vortices). External non-classical electromagnetic fields are coupled to the device and interact with the vortices that circulate the ring. The time evolution of this two-mode fully quantum mechanical system is studied. The effect of the quantum statistics of the photons on the quantum statistics of the vortices is discussed. The entanglement between the two systems is quantified.
\end{abstract}

\section{Introduction}

There has been a lot of interest in the interaction of Josephson devices with microwaves for a long time. The new development in the last ten years [1-4] has been the experimental and theoretical study of mesoscopic Josephson devices (with capacitance lower than $10^{-16} \mathrm{~F}$ ) where quantum phenomena are stronger.

At the same time there have been significant developments in quantum optics. Non-classical electromagnetic fields have been studied extensively both experimentally and theoretically. These fields are carefully prepared in a particular quantum state so that the amount of quantum noise is well defined and the statistics of photons are also well defined.

The purpose of this paper is to present some interdisciplinary work that studies the interaction of mesoscopic Josephson devices interacting with non-classical electromagnetic fields (at gigahertz to terahertz frequencies) $[5,6]$. More specifically, we consider a ring made from a Josephson array in the insulating phase. Vortices circulate this ring with high mobility. The ring contains a dual Josephson junction [7-9] through which the vortices tunnel. This is a fully quantum mechanical system and we study explicitly how the quantum noise of the electromagnetic fields affects the quantum noise of the vortex current. Recent work on vortices can be found in [10-28].

Nonlinear systems have been studied extensively in quantum optics in conjuction with nonlinear optical materials. We note that the sinusoidal nonlinearity of dual Josephson junctions in Josephson arrays is much stronger than the

() IEE, 2001

IEE Proceedings online no. 20010394

DOI: 10.1049/ip-smt:20010394

Paper first received 9th November 2000 and in revised form 19th February 2001

A. Konstadopoulou, J.M. Hollingworth, A. Vourdas and J.F. Ralph are with the Department of Electrical Engineering and Electronics, University of Liverpool, Liverpool L69 3GJ, UK

M. Everitt and T.D. Clark are with the School of Engineering and Information Technology, University of Sussex, Falmer, Brighton BN1 9QT, UK

IEE Proc.-Sci. Meas. Technol., Vol. 148, No. 5, September 2001 polynomial nonlinearity with very small coefficients that describes the nonlinear materials used in quantum optics. The periodicity of the sinusoidal nonlinearity also makes the dual Josephson junctions in Josephson arrays very interesting from a theoretical point of view and requires the development of novel highly nonperturbative methods.

Josephson systems have been used for the production of squeezed microwaves $[29,30]$ and they are good candidates for the development of devices operating at terahertz frequencies, which is the modern tendency in communications. There is also a lot of work currently, for their use as quantum gates in quantum technology and quantum computing [31].

\section{Interaction of Josephson arrays with external non-classical microwaves}

We consider a ring made from an array of Josephson junctions with Coulomb coupling constant $E_{C}$ greater than the Josephson coupling constant $E_{J}$. For those values of the parameters the array is in the insulating phase where vortices move with high mobility and charges are confined. Such rings have been considered experimentally in the context of the Aharonov-Casher effect [32-38]. However, our ring has also a 'dual Josephson junction' [7-9]. This plays a similar role for vortices, to the ordinary Josephson junctions for electron pairs. The 'dual phase' of the vortex wavefunction has a discontinuity $\delta$ along the dual Josephson junction. This is analogous to the Cooper-pair wavefunction in superconducting rings with Josephson junctions, which has discontinuity $\phi$ along the junction.

The centre of the ring contains charge $Q(t)$ induced through coupling with an external source of microwaves which are carefully prepared in a particular quantum state (Fig. 1). Microwaves in various quantum states have been produced experimentally in several laboratories (e.g. $[29,30])$. The system operates at low temperatures $\left(\hbar \Omega_{1}>\right.$ $k_{B} T$ and $\hbar \Omega_{2}>k_{B} T$ ), so that the thermal noise is less than the quantum noise in the microwaves and the device. The dissipation in the system is assumed to be negligible.

The Hamiltonian describing this system contains an inductive term $1 / 2 L\left(I-I_{m w}\right)^{2}$, a capacitive term $\left(Q-Q_{m w}\right.$ $2 C)^{2}$ and a 'dual Josephson' term $E_{d J}(1-\cos \delta)$. Here $L$ and 
$C$ are the inductance and capacitance of the system, respectively. For simplicity we assume that the inductance and capacitance of the ring are equal to the inductance and capacitance of the circuit that produces the microwaves. $I$ and $I_{m w}$ are the total and external (microwave) current respectively, flowing in the radial direction of the device. $Q$ and $Q_{m w}$ are the total and external (microwave) charge, in the inner boundary of the ring. $E_{d J}$ is the dual Josephson coupling constant and $\delta=\phi_{0} Q$ the dual phase. $\phi_{0}=\pi / e$ is the flux quantum (in units where $\hbar=k_{B}=c=1$ ). The sinusoidal term $E_{d}(1-\cos \delta)$ describes vortex tunnelling through the dual Josephson junction. This is analogous to the well-known term $E_{J}(1-\cos \phi)$, which describes the tunnelling of electron pairs in Josephson junctions.

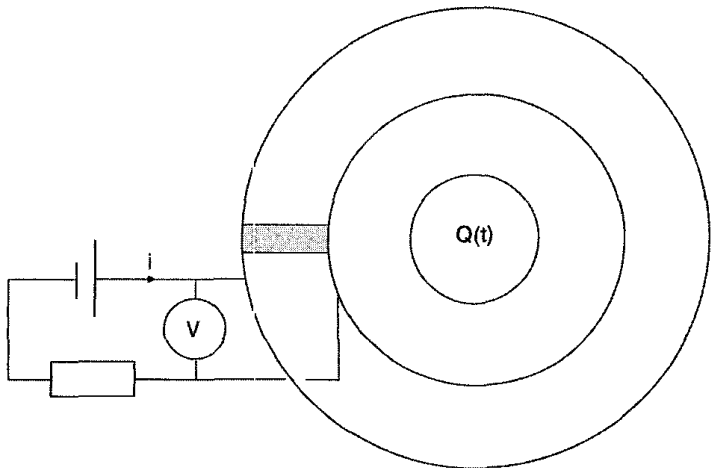

Fig. 1 Josephson array ring in the insulating phase, coupled to a source of non-classical microwaves

The ring contains a dual Josephson junction (i.e. a Josephson junction for vortices). The voltmeter measures the vortex current. The current $i$ compensates the dissipation.

Quantisation of the device is done with the creation and annihilation operators

$$
\begin{aligned}
a_{1} & =\left(\frac{1}{2 \Omega_{1} C}\right)^{1 / 2}\left[Q+i \Omega_{1}^{-1} I\right] \\
a_{1}^{\dagger} & =\left(\frac{1}{2 \Omega_{1} C}\right)^{1 / 2}\left[Q-i \Omega_{1}^{-1} I\right] \\
{\left[a_{1}, a_{1}^{\dagger}\right] } & =1
\end{aligned}
$$

where $\Omega_{1}=(L C)^{-1 / 2}$ is the frequency of the device. We note that this is the frequency of the linear part of the device. The sinusoidal nonlinearity renormalises this frequency (i.e. there is an $a_{1}^{\dagger} a_{1}$ term within the $\cos \delta$ nonlinearity). The electromagnetic field is quantised with the operators:

$$
\begin{aligned}
a_{2} & =\left(\frac{1}{2 \Omega_{2} C}\right)^{1 / 2}\left[Q_{m w}+i \Omega_{2}^{-1} I_{m w}\right] \\
a_{2}^{\dagger} & =\left(\frac{1}{2 \Omega_{2} C}\right)^{1 / 2}\left[Q_{m w}-i \Omega_{2}^{-1} I_{m w}\right] \\
{\left[a_{2}, a_{2}^{\dagger}\right] } & =1
\end{aligned}
$$

where $\Omega_{2}=(L C)^{-1 / 2}$ is the frequency of the microwaves. We note that for the parameters considered $\Omega_{1}=\Omega_{2}$.

The Hamiltonian can now be written as

$$
\begin{aligned}
H= & \Omega_{1} a_{1}^{\dagger} a_{1}+\Omega_{2} a_{2}^{\dagger} a_{2}-E_{d J} \cos \left[\mu\left(a_{1}^{\dagger}+a_{1}\right)\right] \\
& -\Omega_{1}\left(a_{1}^{\dagger} a_{2}+a_{1} a_{2}^{\dagger}\right)
\end{aligned}
$$

where $\mu=\phi_{0}\left(\Omega_{1} C / 2\right)^{1 / 2}$.

\section{Time evolution}

In the absence of dissipation the the density matrix of the system $\rho(t)$ is given by

$$
\rho(t)=\exp [i H t] \rho(0) \exp [-i H t]
$$

where $\rho(0)$ is the density matrix at $t=0$. We have calculated numerically $\rho(t)$ and the reduced density matrices

$$
\rho_{1}(t)=\operatorname{Tr}_{2} \rho(t) ; \quad \rho_{2}(t)=\operatorname{Tr}_{1} \rho(t)
$$

Using the reduced density matrices we calculated the average number of quanta in each mode

$$
\left\langle N_{i}\right\rangle=\operatorname{Tr}\left[a^{\dagger} a \rho_{i}\right]
$$

as functions of time.

The infinite dimensional matrix $\left\langle M_{1}, M_{2}|H| N_{1}, N_{2}\right\rangle$ has been truncated for the numerical calculations, with $M_{1}, N_{1}$ taking values from 0 up to $K_{1 \max }$ and $M_{2}, N_{2}$ taking values from 0 up to $K_{2 \max } . K_{1 \max }$ and $K_{2 \max }$ were taken to be much greater than $\left\langle N_{1}\right\rangle$ and $\left\langle N_{2}\right\rangle$, respectively. As a measure of the accuracy of the approximation we calculated the traces of the truncated matrices. In the limit $K_{1 \max } \rightarrow \infty$ and $K_{2 \max } \rightarrow \infty$ they are equal to 1 ; and in the truncated case they should be very close to 1 . In all our results the above sum was greater than 0.98 .

In Fig. 2 we assume that at $t=0$ the device is in the vacuum state $|0\rangle\left(a_{1}|0\rangle=0\right)$ and the microwaves are in the number state $|N=1\rangle\left(a_{2}^{\dagger} a_{2}|1\rangle=|1\rangle\right)$. The results presented show an exchange of energy between the microwaves and the device.

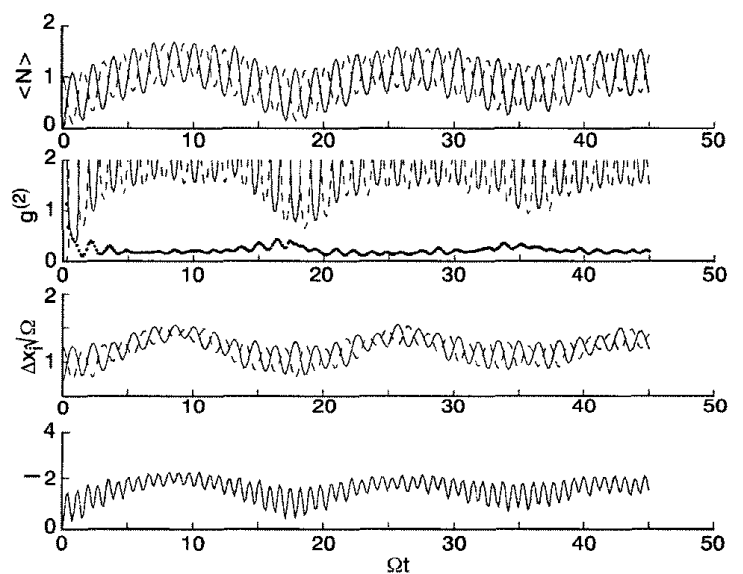

Fig.2 Results showing exchange of energy between microwaves and device At time $t=0$ the device is in the vacuum state $|0\rangle$ and the microwaves in the number state $|N=1\rangle$

$\Omega=\omega=1.5 \times 10^{-4}, E_{d J}=10^{-4}, \mu=2.8408$ and truncation $K_{\text {imax }}=10$ First graph: $\left\langle N_{1}\right\rangle$ (solid line) and $\left\langle N_{2}\right\rangle$ (broken line) as functions of time Second graph: $\mathrm{g}_{11}{ }^{(2)}$ (solid line), $\mathrm{g}_{22}{ }^{(2)}$ (broken line) and $r$ (dotted line) as functions of

Third graph: uncertainties $\Delta x_{1} \sqrt{ } \Omega$ (solid line) and $\Delta x_{2} \sqrt{ } \Omega$ (dotted line) as functions of

Fourth graph: entanglement entropy $I$ (in nats) as a function of time

In Fig. 3 we assume that at $t=0$ the device is in the vacuum state $|0\rangle\left(a_{1}|0\rangle=0\right)$ and the microwaves are in the coherent state $|A=1.5\rangle\left(a_{2}|A\rangle=A|A\rangle\right)$. The results presented show an exchange of energy between the microwaves and the vortices in the device.

The microwaves have been carefully prepared in a quantum state and this implies that the quantum statistics of the photons threading the ring, is known. In our analysis we study a 'quantum Faraday law' and investigate how the quantum statistics and quantum noise of the photons affects the quantum statistics and quantum noise of the tunnelling vortices. To quantify the quantum statistics, we 
present in figures Figs. 2 and 3 the second order correlations

$$
\begin{gathered}
g_{i i}^{(2)}=\frac{\left\langle N_{i}^{2}\right\rangle-\left\langle N_{i}\right\rangle}{\left\langle N_{i}\right\rangle^{2}} ; \quad i=1,2 \\
g_{12}^{(2)}=\frac{\left\langle N_{1} N_{2}\right\rangle}{\left\langle N_{1}\right\rangle\left\langle N_{2}\right\rangle}
\end{gathered}
$$

and the ratio

$$
r=\frac{\left[g_{12}^{(2)}\right]^{2}}{g_{11}^{(2)} g_{22}^{(2)}}
$$

The $g_{11}{ }^{(2)}$ describe vortex bunching or antibunching, and the $g_{22}{ }^{(2)}$ describe photon bunching or antibunching. The quantum noise is quantified with the uncertainties

$$
\left(\Delta x_{i}\right)^{2}=\operatorname{Tr}\left(\rho x_{i}^{2}\right)-\left[\operatorname{Tr}\left(\rho x_{i}\right)\right]^{2} ; \quad i=1,2
$$

which are presented in Figs. 2 and 3.

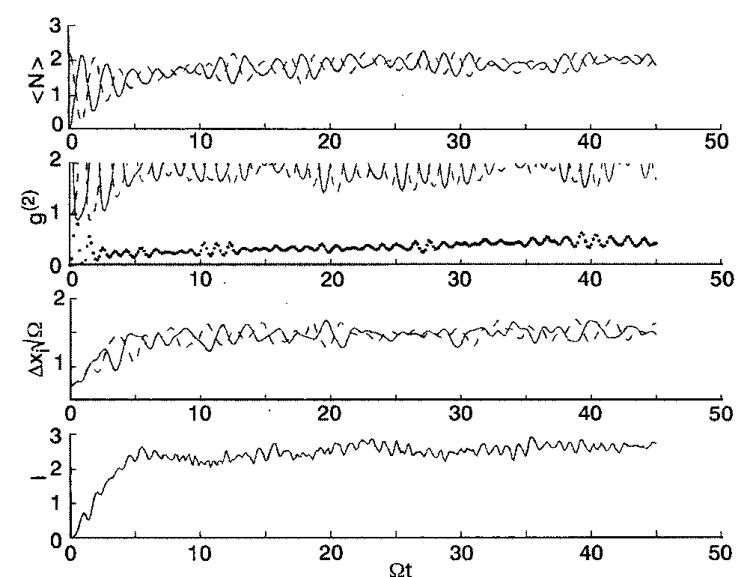

Fig.3 Results showing exchange of energy between microwaves and vortices in the device

At time $t=0$ the device is in the vacuum state $\{0\rangle$ and the microwaves in the coherent state $|A=1.5\rangle$

$\Omega=\omega=1.5 \times 10^{-4}, E_{d J}=10^{-4}, \mu=2.8408$ and truncation $K_{\text {inax }}=10$ First graph: $\left\langle N_{1}\right\rangle$ (solid line) and $\left\langle N_{2}\right\rangle$ (broken line) as functions of time
Second graph: $g_{11}{ }^{(2)}$ (solid line), $g_{22}{ }^{(2)}$ (broken line) and $r$ (dotted line) as functions of

Third graph: uncertainties $\Delta x_{1} \sqrt{ } \Omega$ (solid line) and $\Delta x_{2} \sqrt{ } \Omega$ (dotted line) as functions of time

Fourth graph: entanglement entropy $I$ (in nats) as a function of time

At $t=0$ the system is not entangled, but as time evolves the microwaves entangle with the Josephson array device. As a measure of this entanglement we have calculated the entanglement entropy [39-42]

$$
I=S\left(\rho_{1}\right)+S\left(\rho_{2}\right)-S(\rho)
$$

where $S=-\operatorname{Tr} \rho \ln \rho$ is the von Neumann entropy. The entanglement entropy $I$ is positive according to the subadditivity property. The results show that although originally the system was disentangled, it becomes strongly entangled later.

\section{Discussion}

We have considered a ring made from a Josephson array in the insulating phase. The ring contains a dual Josephson junction which is described mathematically with a sinusoidal nonlinear term. External non-classical electromagnetic fields are coupled to the device and interact with the vortices that circulate the ring.

We have calculated the time evolution of this fully quantum mechanical two-mode system. The results have shown the exchange of energy between the electromagnetic field and the vortices. We have also shown quantitatively (with the $g^{(2)}$ ) how the quantum noise of the electromagnetic field affects the quantum noise of the vortices, and how the two modes become entangled. The calculations have ignored dissipation and work is in progress in the direction of assessing the effect of dissipation [43].

The results can be useful in the context of quantum gates based on Josephson technology; and also in the context of terahertz technology.

\section{Acknowledgments}

J. M. Hollingworth and A. Konstadopoulou gratefully acknowledge support from the Engineering and Physical Sciences Research Council.

\section{References}

1 SCHON, G., and ZAIKIN, A.D.: 'Quantum coherent effects, phase transitions, and the dissipative dynamics of ultra small tunnel junctions', Phys. Rep., 1990, 198, pp. $237-412$

2 KASTNER, M.A.: 'The single-electron transistor', Rev. Mod. Phys., 1992,64 , pp. $849-858$

3 SPILLER, T.P CLARK, T.D, PRANCE, R.J., and WIDOM, A. 'Quantum phenomena in circuits at low temperatures', Prog. Low Temp. Phys., 1992, 13, p. 219

4 GRABERT, H., and DEVORET, M.H. (Eds.): 'Single-charge tunneling'. NATO ASI series, 294 (Plenum, NY, 1992)

5 VOURDAS, A.: 'Mesoscopic Josephson junctions in the presence of nonclassical electromagnetic fields', Phys. Rev. B, Condens. Matter, 1994,49 , pp. $12040-12046$

6 VOURDAS, A., and SPILLER, T.P.: 'Quantum theory of the interaction of Josephson junctions with non-classical microwaves', $Z$. Phys. B, Condens. Matter, 1997, 102, pp. 43-54

7 WIDOM, A., MEGALOUDIS, G., CLARK, T.D., PRANCE, R.J., and PRANCE, H.: 'Quantum electrodynamic charge space energy bands in singly connected superconducting weak links', J. Phys. A, Math. Gen., 1982, 15, pp. 3877-3879

8 POULTON, D.A.: 'Quantum circuit behaviour'. DPhil. thesis, University of Sussex, 1989

9 VOURDAS, A.: 'Dual Josephson phenomena with vortices', Europhys. Lett., 1999, 48, pp. 201-207

10 VAN DER ZANT, H.S.J., FRITSCHY, F.C., ORLANDO, T.P., and MOOIJ, J.E.: 'Ballistic vortices in Josephson-junction arrays', Europhys. Lett., 1992, 18, pp. 343-348

11 VAN OUDENAARDEN, A., and MOOIJ, J.E.: 'One-dimensional Mott insulator formed by quantum vortices in Josephson junction arrays', Phys. Rev. Lett., 1996, 76, pp. 4947-4950

12 VAN OUDENAARDEN, A., VARDY, S.J.K., and MOOIJ, J.E.: 'One-dimensional localization of quantum vortices in disordered Josephson junction arrays', Phys. Rev. Lett. 1996, 77, pp. 4257-4260

13 CHUNG, J.S., LEE, K.H., and STROUD, D.: 'Dynamical properties of superconducting arrays', Phys. Rev. B, Condens. Matter, 1989, 40, pp. $6570-6580$

14 OCTAVIO, M., FREE, J.U., BENZ, S.P., NEWROCK, R.S., MAST, D.B., and LOBB, C.J.: 'Simulations and interpretation of fractional giant Shapiro steps in two-dimensional Josephson-junction arrays', Phys. Rev. B. Condens. Matter, 1991, 44, pp. 4601-4609

15 SOHN, L.L., RZCHOWSKI, M.S., FREE, J.U., TINKHAM, M., and LOBB, C.J.: 'Effect of current direction on the dynamics of Josephson-junction arrays', Phys. Rev. B, Condens. Matter, 1992, 45, pp. 3003-3012

16 LARKIN, A.I., OVCHINNIKOV, YU.N., and SCHMID, A.: 'Quantum creep of vortices in granular superconductors', Physica B, $1988, \mathbf{1 5 2}$, pp. 266-281

17 ECKERN, U., and SCHMID, A.: 'Quantum vortex dynamics in granular superconducting films', Phys. Rev. B, Condens. Matter, 1989, 39. pp. $6441-6454$

18 FALO, F., BISHOP, A.R., and LOMDAHL, P.S.: 'I-V characteristics in two-dimensional frustrated Josephson-junction arrays', Phys. Rev B. Condens Matter. 1990, 41, pp. 10983-10993

19 FAZIO, R., and SCHON, G.: 'Charge and vortex dynamics in arrays of tunnel junctions', Phys. Rev. B, Condens. Matter, 1991, 43, pp. $5307-5320$

20 BENZ, S.P., and BURROUGHS, C.J.: 'Coherent emission from twodimensional Josephson junction arrays', Applied Phys. Lett., 1991, 58, pp. 2162-2164

21 USTINOV, A.V., DODERER, T., MAYER, B., HUEBENER, R.P., GALUBOV, A.A., and OBBOZNOV, V.A.: 'Experimental study of the interaction of fluxons with an Abrikosov vortex in a long Josephson junction', Phys. Rev. B, Condens. Matter, 1993, 47, pp. 944 956

22 LACHENMAUN, S.G., DODERER, T., HOFFMANN, D., HUEBENER, R.P., BOOI, P.A.A., and BENZ, S.P.: 'Observation of vortex dynamics in two-dimensional Josephson-junction arrays', Phys. Rev. B, Condens. Matter, 1994, 50, pp. 3158-3164 
23 SOHN, L.L., TUOMINEN, M.T., RZCHOWSKI, M.S., FREE, J.U., and TINKHAM, M.: 'AC and DC properties of Josephsonjunction arrays with long-range interaction', Phys. Rev. $B$. Condens. junction arrays with long-range

24 FRANZ, M., and TEITEL, S.: 'Vortex-lattice melting in two-dimensional superconducting networks and films', Phys. Rev. B, Condens. Matter, 1995, 51, pp. $6551-6574$

25 KOSHELETS, V.P., SHITOV, S.V., SHCHUKIN, A.V., FILIPPENKO, L.V., MYGIND, J., and USTINOV, A.V: 'Self-pumping effects and radiation linewidth of Josephson flux-flow oscillators', Phys. Rev. B, Condens. Matter, 1997, 56, pp. 5572-5577

26 USTINOV, A.V., MALOMED, B.A., and SAKAI, S.: 'Bunched fluxon states in one-dimensional Josephson-junction arrays', Phys. Rev. B, Condens. Matier, 1998, 57, pp. 11691-11697

27 BARBARA, P., CAWTHORNE, A.B., SHITOV, S.V., and LOBB C.J.: 'Stimulated emission and amplification in Josephson junction arrays', Phys. Rev. Leit., 1999, 82, pp. 1963-1966

28 CAWTHOME, A.B. BARBARA, P., SHITOV, S.V., LOBB, C.J. WIESENFELD, K., and ZANGWILL, A.: 'Synchronized oscillations in Josephson junction arrays: The role of distributed coupling', Phys. Rev. B, Condens. Matter, 1999, 60, pp. 7575-7578

29 YURKE, B., KAMINSKY, P.G., MILLER, R.E., WHITTAKER, E.A., SMITH, A.D., SILVER, A.H., and SIMON, R.W.: 'Observation of $4.2 \mathrm{~K}$ equilibrium-noise squeezing via a Josephson-parametric amplifier', Phys. Rev Lett, 1988 60 pp. 764-767

30 YURKE B CORRUCCINI, L. R KAMINSKY, P.G., RUPP L.W., SMITH, A.D., SILVER, A.H., SIMON, R.W., and WHITTAKER, E.A.: 'Observation of parametric amplification and deamplification in a Josephson parametric amplifier', Phys. Rev. A, Gen Phys.,39, pp. 2519-2533

31 ORLANDO, T. MOOJ JE TIAN L VAN DER WAL C.H LEVITOV, L.S., LLOYD, S., and MAZO, J.J.: 'Superconducting persistent-current qubit', Phys. Rev. B, Condens. Matter, 1999, 60, pp.
, persistent-curr
$15398-15413$
32 AHARONOV, Y, and CASHER, A.: 'Topological quantum effects for neutral particles', Phys. Rev. Lett. 1984, 53, pp. 319-32

33 REZNIK, B., and AHARONOV, Y.: 'Question of the nonlocality of the Aharonov-Casher effect', Phys. Rev. D, Part. Fields, 1989, 40, pp. $4178-4183$

34 GOLDHABER, A.S.: 'Comment on 'Topological quantum effects for neutral particles', Phys. Rev. Lett., 1989, 62, pp. 482

35 VAN WEES, B.J.: 'Aharonov-Bohm-type effect for vortices in Josephson-junction arrays', Phys. Rev. Lett., 1990, 65, pp. 255-258

36 VAN WEES, B.J.: 'Duality between Cooper-pair and vortex dynamics in two-dimensional Josephson-junction arrays', Phys. Rev. B. Condens. Matter, 1991, 44, pp. 2264-2267

37 ORLANDO, T.P., and DELIN, K.A.: 'Voltage quantization by ballistic vortices in two-dimensional superconductors', Phys. Rev. B, Condens. Matter, 1991, 43, pp. 8717-8720

38 ELION, W.J., WACHTERS, J.J., SOHN, L.L., and MOOIJ, J.E.: 'Observation of the Aharonov-Casher effect for vortices in Josephsonjunction arrays', Phys Rev Lett. 1993, 71, pp. 2311-2314

39 LINDBLAND, G.: 'Entropy, information and quantum measurements', Commun. Math. Phys., 1973, 33, pp. 305-322

40 WEHRL, A.: 'General properties of entropy', Rev. Mod. Phys., 1978, 50, pp. 221-260

41 BARNETT, S.M., and PHOENIX, S.J.D.: 'Information theory, squeezing, and quantum correlations', Phys. Rev. A, At. Mol. Opt Phys., 1991, 44, pp. 535-545

42 VOURDAS, A.: 'Generalized squeezing, Bogoliubov quasiparticles, and information in two- and three-mode systems', Phys. Rev. A, At. Mol. Opt. Phys., 1992, 46, pp. 442 451

43 LEGGETT, A.J., CHAKRAVARTY, S., DORSEY, A.T., FISHER, P.A., GARG, A., and ZWERGER, W.: 'Dynamics of the dissipative two-state system', Rev. Mod. Phys., 1987, 59, pp. 1-85 